
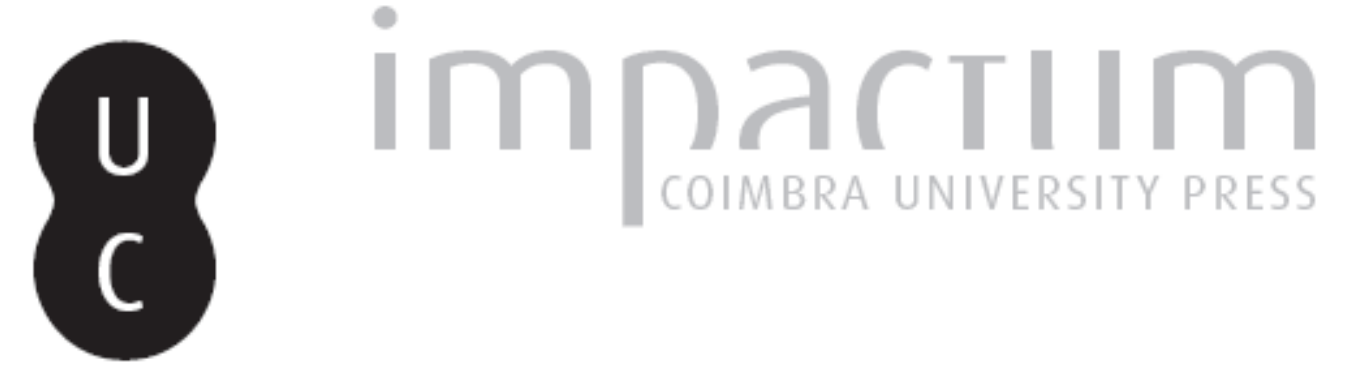

\title{
Le commerce de la vérité: économie et commerce dans les Lois de Platon
}

\section{Autor(es): Helmer, Etienne}

Publicado por: Imprensa da Universidade de Coimbra

URL persistente:

URl:http://hdl.handle.net/10316.2/43595

DOI:

DOI:https://doi.org/10.14195/2183-4105_17_3

Accessed : $\quad$ 26-Apr-2023 09:38:18

A navegação consulta e descarregamento dos títulos inseridos nas Bibliotecas Digitais UC Digitalis, UC Pombalina e UC Impactum, pressupõem a aceitação plena e sem reservas dos Termos e Condições de Uso destas Bibliotecas Digitais, disponíveis em https://digitalis.uc.pt/pt-pt/termos.

Conforme exposto nos referidos Termos e Condições de Uso, o descarregamento de títulos de acesso restrito requer uma licença válida de autorização devendo o utilizador aceder ao(s) documento(s) a partir de um endereço de IP da instituição detentora da supramencionada licença.

Ao utilizador é apenas permitido o descarregamento para uso pessoal, pelo que o emprego do(s) título(s) descarregado(s) para outro fim, designadamente comercial, carece de autorização do respetivo autor ou editor da obra.

Na medida em que todas as obras da UC Digitalis se encontram protegidas pelo Código do Direito de Autor e Direitos Conexos e demais legislação aplicável, toda a cópia, parcial ou total, deste documento, nos casos em que é legalmente admitida, deverá conter ou fazer-se acompanhar por este aviso.

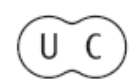


ISSN 2079-7567 eISSN 2183-4105

0

\section{Established 1989}

http://platosociety.org/

\section{Papers}

Cristina lonescu Elenchus, Recollection, and the Method of Hypothesis in the Meno

Lucas Soares

La relectura positiva de la tradición poética griega en el Banquete de Platón

Etienne Helmer Le commerce de la vérité: économie et commerce dans les Lois de Platon

Alan Pichanick

Socratic Silence in the Cleitophon

Thanassis Gkatzaras The Form of the Good in Plato's Timaeus

James M. Ambury Dialectical Epimeleia:

Platonic Care of the Soul and Philosophical Cognition
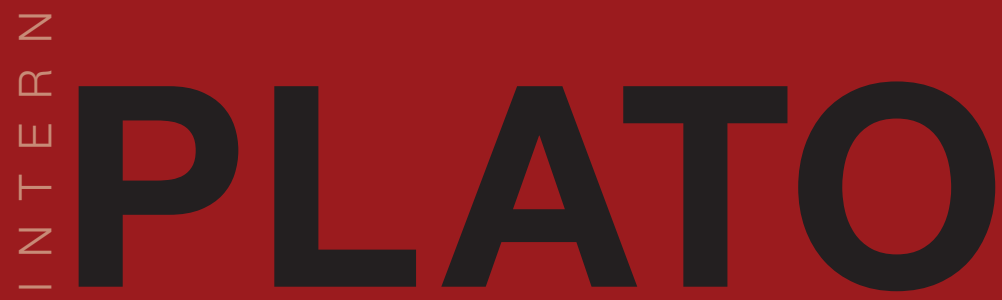

Book Reviews

Nicholas Zucchetti

Essays on Plato's Epistemology by Franco Trabattoni

Alan Pichanick

Socrates and Self-Knowledge by Christopher Moore
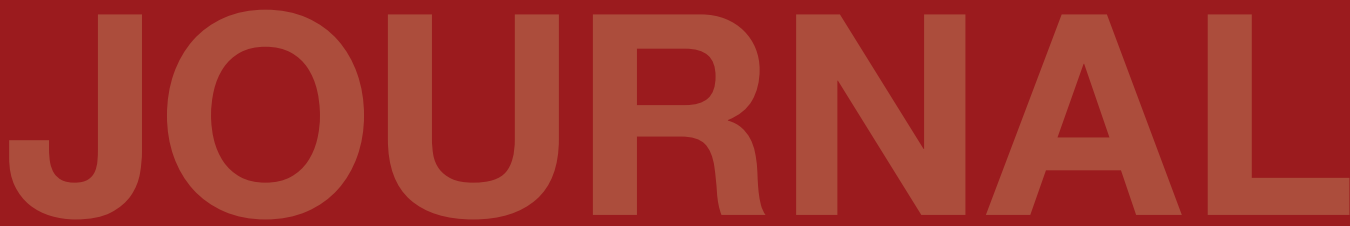

Société Platonicienne Internationale

Associazione Internazionale dei Platonisti

Sociedad Internacional de Platonistas

Internationale

Platon-Gesellschaft

Imprensa da

Universidade

de Coimbra

Coimbra

Universiy

Press 


\section{Le commerce de la} vérité: économie et commerce dans les Lois de Platon ${ }^{1}$

\author{
Etienne Helmer \\ etiennehelmer@hotmail.fr \\ University of Puerto Rico
}

\section{ABSTRACT}

Unlike the accepted idea that Plato's view of trade and business is totally negative, my claim is that the whole set of limitations the Athenian prescribes to trade in the Laws only aims at minimizing its bad effects. Far from completely disapproving trade in itself, Plato acknowledges it has a practical positivity. Besides the needs, which are the first and most evident ends of trade and business, Plato shows that these activities also have to do with truth and justice, and can fulfill a truly positive moral and political function in the city of Magnesia under certain conditions.

Keywords: business, Laws, money, Plato, trade, truth.

\section{INTRODUCTION}

On prête souvent à Platon un préjugé hostile aux activités mercantiles tant intérieures qu'extérieures, en particulier dans les Lois. À l'appui de cette idée, on invoque, pour le commerce avec les cités étrangères, l'idéal d'autarcie que les Lois paraissent viser, ainsi que le danger de corruption que ce type de négoce fait peser sur les mœurs de la cité ; et, pour le commerce intérieur, la condamnation sans appel prononcée par l'Athénien contre l'avidité matérielle que ces échanges favorisent. ${ }^{2}$

Pourtant, on note souvent aussi que le portrait du commerce dans ce dialogue n'est pas complètement négatif. L’Athénien incrimine davantage une certaine pratique du commerce - sa pratique 'financière', celle qui ne vise que le profit et dont la forme privilégiée est la revente au détail ( $\kappa a \pi \eta \lambda \varepsilon i ́ a)$ d'un produit acheté en gros - que le commerce lui-même. Il se livre même à un éloge de la vente au détail (XI, 918a) qu'il qualifie de 'bienfaitrice' ( $\left.\varepsilon \dot{\varepsilon} \varepsilon \rho \gamma^{\varepsilon} \tau \eta \varsigma, 918 b\right)$ quand elle est pratiquée conformément à ce qu'elle est par nature, à savoir quand, grâce à la monnaie, elle introduit de la commensurabilité entre des biens qui en sont de prime abord dépourvus (XI, 918a-c; d-e).

Néanmoins, même les commentateurs des Lois qui reconnaissent cette positivité aux activités commerciales ne leur rendent pas complètement justice, faute de voir deux choses: d'une part, que le commerce n'est qu'un cas particulier des pratiques tournées vers l'acquisition d'argent, qui sont précisément celles que l'Athénien écarte le plus possible de la cité des Magnètes; d'autre part, que les enjeux du commerce dans ce dialogue dépassent de loin les questions économiques. Ainsi en va-t-il dans un article de S. Sauvé-Meyer, le seul qui, à ma connaissance, se livre à un examen détaillé de ces passages des Lois. ${ }^{3}$ Si l'auteure note à juste 
titre le double portrait brossé du commerce par l'Athénien - il est à la fois déprécié et loué - elle ne s'étonne pas, en revanche, de la valorisation étonnamment très positive dont il fait l'objet quand il est bien effectué. La preuve en est dans le choix des extraits qu'elle examine. À propos du passage XI, 918a8-c3, son commentaire est le suivant: 'Le négoce (spécifiquement l'échange de marchandises contre de l'argent) a la fonction bénéfique d'égaliser et de faciliter la distribution des ressources (918a).' Elle poursuit un peu plus loin en ces termes: 'La véritable fonction du kapèlos n'est pas de maximiser son propre profit par l'échange, mais plutôt de pourvoir aux besoins de la communauté en facilitant la distribution convenable des ressources. ${ }^{4}$ Tout ceci est exact: la recherche du profit est une pratique altérée des activités économiques en général, et des activités commerciales en particulier dont la véritable fonction est d'assurer une 'distribution convenable des ressources' grâce à la monnaie comme moyen de mesure. Mais le texte qui suit ce passage, et que l'auteure ne cite pas et ne commente pas, laisse attendre du commerce bien plus qu'une simple 'distribution convenable des ressources'. L’Athénien prononce en effet à son sujet un véritable éloge, quand il est correctement accompli:

Supposons [...] qu'une prescription contraigne pendant un certain temps les hommes qui en tout lieu sont les meilleurs de tenir auberge, d'ouvrir boutique ou d'exercer quelque activité de cette sorte [...], nous saurions alors quelle affection et

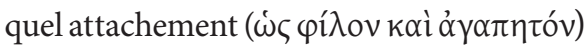
méritent chacune de ces professions et nous aurions pour elles toutes, qui seraient pratiquées selon la règle et dans une intégrité incorruptible, la révérence qu'on a pour une mère et pour une nourrice. Lois XI, 918e
Ce qui échappe aux commentateurs de cette section, c'est que la forte suspicion et les limites étroites pesant sur les activités commerciales dans le reste des Lois sont précisément à la mesure de l'importance symétrique que l'Athénien leur reconnaît quand elles sont bien exercées. Derrière les objets échangés, qu'il s'agisse de biens ou de services, Platon perçoit qu'il est moins question de satisfaction économique que de vérité et de justice, au sens où ce qui circule dans les transactions commerciales, c'est aussi et peut-être avant tout une relation éthique et politique. Les mesures qu'il propose pour encadrer les pratiques commerciales n'ont pas seulement pour objet d'assurer les conditions d'un commerce juste parce qu'exempt de fraude, mais de garantir la circulation de la vérité au service de la justice à l'un des niveaux les plus élémentaires et fondamentaux des échanges ayant cours dans la cité. Le commerce dans la cité des Lois est, c'est ma thèse, un commerce de la vérité.

Pour le montrer, j'exposerai d'abord les motifs pour lesquels l'Athénien préconise de limiter les échanges commerciaux, en soulignant que la défiance qu'il leur témoigne vise moins le commerce lui-même qu'une économie 'financière' tournée vers l'acquisition d'argent et dans laquelle le commerce jouerait un rôle essentiel. Je montrerai ensuite quelles mesures législatives il propose pour encadrer un commerce certes réduit mais inévitable dans la cité. Enfin, je conclurai en montrant pourquoi l'enjeu de ces mesures ne se limite pas à l'économie mais a pour objet la vérité et, par elle, la justice. Avant d'y venir, précisons que le grec ne dispose pas d'un terme général désignant ce que nous entendons par 'commerce', à savoir des transactions d'achat et de vente, principalement monétaires, et destinées à dégager un profit ou un bénéfice. Il serait plus juste de parler de 'transactions commerciales' ou 
d' 'échanges commerciaux' pour lesquels le grec emploie une multiplicité de mots, selon la voie de commerce adoptée (par terre ou par mer), et selon que le négoce se fait en gros ou au détail. ${ }^{5}$ À quoi il faut ajouter que le sens de ces mots n'est pas totalement fixe, et que ni la recherche d'un bénéfice ni la dimension monétaire ne sont nécessairement présentes dans ces transactions. ${ }^{6}$ Ces précautions terminologiques prises, je parlerai néanmoins de 'commerce' par commodité.

\section{LIMITER LE COMMERCE, LIMITER LE DÉSIR D'ARGENT}

Dans le projet de colonie que l'Athénien élabore avec Clinias et Mégille, tout signale une claire intention de limiter les activités commerciales avec l'étranger, afin de créer le contexte le plus favorable pour acquérir la vertu et pour que la cité ne dépende le plus possible que d'elle-même. C'est ce qui apparaît à la fois dans les considérations géographiques relatives au choix du territoire de cette colonie, ainsi que dans son orientation économique générale.

\section{La géographie de la cité contre la tentation du commerce (IV, 704b-705b)}

Le commerce de la cité que Clinias a la charge de fonder pour y établir une colonie de Cnossos est limité d'abord par la géographie des lieux, présentée au livre IV. Précisons que c'est une géographie 'politique': ce qui pourrait passer pour du déterminisme matériel est plutôt ici à entendre comme un ensemble de déterminations ouvrant vers des possibles. La nature du territoire envisagé pour cette colonie ne pèse pas de tout son poids sur son avenir, puisque son exploitation et son organisation dépendent aussi, et sans doute d'abord, de conditions politiques et morales, en particulier de l'idée du bien à laquelle les interlocuteurs sont arrivés dans les livres antérieurs. Ce bien, c'est l'unité de la polis, obtenu par des lois qui se préoccupent de toutes les vertus et de leur juste hiérarchie (I, 625c-628e).

Située à environ quatre-vingts stades de la mer (soit environ $14 \mathrm{~km}$ ), la cité produit presque tout ce qu'il lui faut, si bien qu'elle 'ne manque

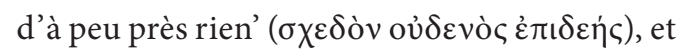
elle n'a pas de cité voisine proche (704c). Tout, dans ce portrait géographique et dans l'analyse qu'en propose ensuite l'Athénien, traduit de sa part de fortes réserves vis-à-vis du commerce avec l'extérieur. À commencer par son 'Quoi?

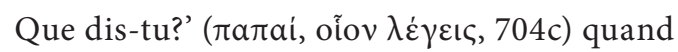
il apprend de Clinias que la cité a 'les meilleurs ports [s.e. naturels] qu'on puisse avoir' (704b): l'Athénien voit un grave défaut là où Clinias voit un grand avantage. Sur le ton de la tragédie, son exclamation traduit son inquiétude devant une situation qui risquerait de coûter le bien politique à cette nouvelle cité, si elle n'était compensée par d'autres avantages, notamment celui de produire presque tout ce qu'il lui faut.

Dans le bilan qu'il dresse des probabilités que la future cité a d'atteindre ou non la vertu étant données ces conditions géographiques, il expose ensuite les raisons de ses réserves à l'égard du commerce avec l'extérieur:

En effet, si elle devait être au bord de la mer, avec de bons ports, si elle ne produisait pas tout ce qu'il lui faut et si elle devait manquer de beaucoup de choses, [la cité] aurait besoin d'un grand sauveur pour éviter d'offrir une multitude de mœurs aussi bigarrées que perverses

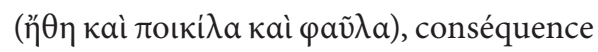
naturelle d'une telle situation. En fait ces quatre-vingts stades apportent une consolation. À vrai dire, notre cité est située 
plus près de la mer qu'il ne le faudrait, dans la mesure où tu la dis pourvue de bons ports [705a]; cependant, il y a aussi de quoi s'en féliciter. Pour un territoire, la proximité de la mer agrémente la vie de tous les jours; mais en définitive, c'est un "voisinage bien saumâtre". Car en le remplissant de négoce et de business réalisé par la vente au détail ( $\dot{\varepsilon} \mu \pi \mathrm{r}$ pias

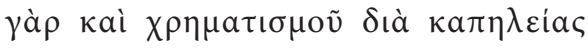
$\dot{\varepsilon} \mu \pi \iota \mu \pi \lambda \tilde{\alpha} \sigma \alpha$ aủंท́v), et en implantant dans les âmes des mœurs instables et

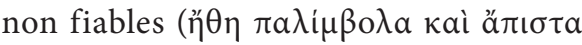

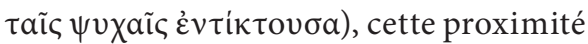
fait que la cité manque de confiance et

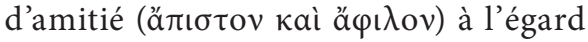
d'elle-même, aussi bien qu'à l'égard des autres hommes. En la matière, on peut certes trouver une consolation dans le

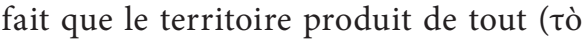
$\pi \alpha \dot{\mu} \varphi о \rho о \varsigma)$ [705b], même si le fait d'être accidenté l'empêche évidemment de produire à la fois de tout et en grande quanti-

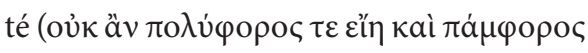

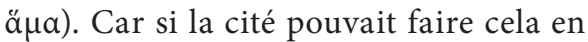
même temps, elle exporterait beaucoup ( $\pi \mathrm{o} \lambda \lambda \grave{\eta} \nu \dot{\varepsilon} \xi \alpha \gamma \omega \gamma \eta \dot{\eta} v)$, ce qui emplirait ses coffres de monnaie d'argent et d'or; et [...] c'est là ce qui engendre pour ainsi dire la pire des calamités pour une cité qui doit acquérir des mœurs nobles et justes.

\section{Lois IV, 704d-705b}

Ce passage décrit une hypothèse et ses conséquences: les effets moraux et politiques négatifs que le commerce avec l'extérieur aurait sur une cité dont il serait la principale ressource économique pour se procurer le nécessaire. Les effets moraux, tout d'abord, consisteraient en un manque d'unité des mœurs, la bigarrure faisant écho au portrait de la démocratie dans la République, régime de la multiplicité plutôt que de l'unité.? Chacun y mène la vie qu'il souhaite, sans souci d'aucune valeur commune. Quant au caractère pervers ou mauvais ( $\varphi \alpha \tilde{v} \lambda \alpha)$ que le commerce insufflerait aux mœurs, il tiendrait probablement au fait, souvent noté, que le commerce se prête particulièrement bien à la tromperie - ce qui ne signifie pas qu'il l'implique toujours. ${ }^{8}$ Quand elle a lieu, cette tromperie n'est pas forcément intentionnelle: s'il n'est pas aussi médecin ou maître de gymnastique, le revendeur de nourriture, en gros ou au détail, ignore si son produit est bon ou mauvais pour le corps, et il en va de même pour le client (Protagoras 313c-d). Le commerce est, en tant que commerce, un art ignorant: ni vrai ni faux, il peut faire passer, au propre comme au figuré, le faux pour le vrai. Le soupçon qui pèse par nature sur lui, notamment aux yeux d'une certaine aristocratie, provient de la puissance d'illusion dont il est capable. ${ }^{9}$ C'est pour cette raison que Platon dépeint toujours les sophistes en commerçants avant d'entrer dans le détail de leurs théories. ${ }^{10}$ Selon lui, ils exploitent cette ignorance inhérente au commerce en tant que commerce pour écouler du faux et de l'injustice sous des dehors de vérité et de justice. Dans notre passage des Lois, l'Athénien souligne que le risque de tromperie inhérent au commerce introduit dans les âmes des mœurs instables et par là peu fiables. L'appât du gain ( $\chi \rho \eta \mu \alpha \tau ı \sigma \mu o \tilde{)})$ peut en effet conduire à des pratiques commerciales douteuses. Trois lois sur le commerce proposées plus tard par l'Athénien en donnent indirectement une idée (XI, 917b-d): il est interdit de vendre une même marchandise à deux prix différents le même jour, de vendre des produits falsifiés, et de faire l'éloge (ë́raıvoc, 917c) de sa marchandise. Dans les trois cas, 
le commerce met en jeu une éthique de la vérité, qui se trouve menacée par les pratiques malhonnêtes visées dans ces lois. En effet, qu'une même marchandise soit vendue le même jour à deux prix distincts laisse planer un doute sur son 'vrai' prix et sur l'intégrité du vendeur, sans compter le préjudice causé à l'égalité entre les clients. L'objet falsifié est, bien sûr, la tromperie par excellence, où le faux-semblant se donne pour ce qu'il n'est pas. Enfin, l'éloge dans sa pratique la plus ordinaire repose sur l'ignorance de son objet, et entend 'le doter de tous les attributs, pour proclamer l'excellence de sa nature, de façon à faire apparaître qu'il est le plus beau et le meilleur possible - aux ignorants, cela va sans dire, mais pas en tout cas [...] à ceux qui savent à quoi s'en tenir' (Banquet 199a). ${ }^{11}$ Quand il repose sur l'ignorance et la rhétorique, l'éloge peut faire passer un âne pour un cheval et, cas plus grave, le mal pour le bien (Phèdre 260b-d).

Ainsi pratiqué, le commerce ruine ensuite les relations les plus élémentaires dans la cité, hors de la sphère domestique. Une économie principalement marchande affecte l'unité de la polis en la privant de cette amitié ( $p$ hilia) et de cette confiance indispensables pour souder les citoyens entre eux. ${ }^{12}$ Cette cité n'est plus une, c'est un agrégat d'individus qui se méfient les uns des autres, et ce sentiment se tourne aussi contre 'le reste des hommes', c'est-à-dire les étrangers. Le commerce, on le verra, n'est jamais étranger à la guerre chez Platon.

La cité des Lois aura cependant de grandes chances d'échapper à ces travers, grâce à deux limites de nature géographique, qui s'appliquent respectivement aux importations et aux exportations, avec comme conséquence mécanique une limitation du commerce de détail. Ce qui sauve la future colonie des importations massives, c'est que

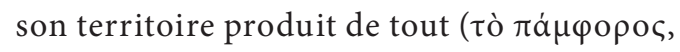
704d; 705b): la diversité lui évite d'importer, du moins d'importer beaucoup. ${ }^{13}$ Mais cette première limite géographique pourrait devenir un danger, si elle n'était elle-même limitée quantitativement: couplée à l'abondance ( $\pi \circ \lambda \dot{v} \varphi \circ \rho \circ \varsigma, 705 \mathrm{~b}$ ), la diversité risquerait d'alimenter un appétit de richesse dont, l'Athénien l'a montré, il ne sort en général rien de bon, ni à l'intérieur de la cité ni dans ses rapports avec les autres cités. ${ }^{14}$ Fort heureusement, la production n'atteint pas de grandes quantités - ce qui empêche de grandes exportations tout en suffisant au nécessaire.

La géographie freine donc ce que l'Athénien semble considérer comme la 'tentation du commerce', ou plus exactement, la tentation d'exploiter l'ignorance inhérente au commerce à des fins de tromperie et de violence, dans l'idée, couramment admise mais fausse aux yeux de Platon, que la richesse serait le critère de la puissance individuelle et collective. ${ }^{15}$

Acquérir le nécessaire ou acquérir de l'argent: la question des médiations (VIII, $842 c-d)$

Dans la continuité de cette géographie, l'Athénien dessine au livre VIII des Lois l'orientation générale de l'économie de la future colonie. Elle consistera à acquérir le nécessaire à partir des ressources très variées du territoire, et avec le moins de médiations possible, au détriment d'une économie 'financière', c'est-à-dire médiatisée par des activités permettant d'acquérir de l'argent ou des revenus pour se procurer, par commerce interposé, 'l'équipement de la

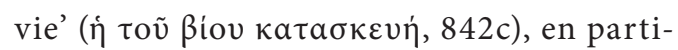
culier 'l'approvisionnement en nourriture' 


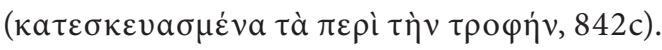
Ce passage marque néanmoins une avancée par rapport au précédent car le critère auquel recourt l'Athénien pour séparer les activités économiques légitimes des autres se précise. Il n'oppose pas, on va le voir, la 'production' au 'commerce': appliquée telle qu'elle, cette dichotomie moderne manque l'essentiel de ce passage où l'exploitation des mines est rangée dans la même catégorie que le négoce maritime ou l'hôtellerie. L'Athénien n'oppose pas une cité productrice à une cité marchande, mais deux manières de se procurer le nécessaire dont l'une, totalement médiatisée par l'argent, a le dangereux pouvoir de faciliter la recherche de ce qui excède le nécessaire.

En effet, en Grèce, 'les ressources des autres cités peuvent être de toutes sortes et de multiples origines' (842c), et elles disposent en général du double de ce dont disposera la cité à venir, car leur approvisionnement en nourriture provient de la mer et de la terre, tandis que la future colonie se contentera d'exploiter sa terre (842c). La référence à la mer est une allusion au commerce, notamment à l'importance qu'il joue à Athènes pour l'approvisionnement en blé, ${ }^{16}$ mais c'est aussi une allusion à la guerre, ces deux pratiques étant souvent liées dans le monde antique en général, et dans l'esprit de Platon en particulier. ${ }^{17}$ Déjà au livre IV, immédiatement après le passage sur le commerce commenté plus haut, l'Athénien se réjouissait que le territoire de la future cité fût vierge d'arbres pour la construction de navires de guerre (IV, 705c-707d), comme si la suite naturelle du commerce extérieur était de livrer la guerre pour accaparer les ressources des ennemis. Les motivations économiques de la guerre doivent donc s'éteindre dès l'instant que l'approvisionnement de la cité ne dépend que d'elle-même, ce que les conditions géo- graphiques doivent en grande partie rendre possible - il y faudra aussi la vertu entière des citoyens (IV, 705e-706a).

L’Athénien énonce ensuite la conséquence d'un tel choix économique pour la cité future: Aussi bien cela rendra plus facile la tâche du législateur car il lui suffira par ailleurs non seulement de moitié moins de lois, mais de beaucoup moins encore, et de plus ce seront des lois convenant plus à des

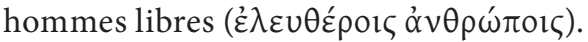
Les affaires de négoce maritime, de gros négoce et de commerce de détail, d'hôtellerie ou de perception d'impôt, de mines, de prêts à intérêt, d'intérêts composés, et des milliers d'autres affaires de cette sorte, le législateur en est libéré et leur dit au revoir. Mais c'est aux agriculteurs et aux pâtres, aux éleveurs d'abeilles, à ceux qui préservent et supervisent leur outillage qu'il donnera des lois.

\section{Lois VIII, 842c-d}

L'allègement du travail législatif résultant du refus d'une économie 'financière' au profit d'une économie tournée vers l'acquisition plus immédiate du nécessaire se fera au bénéfice des 'hommes libres': en quoi on peut entendre soit qu'ils seront ainsi 'libres des liens du commerce', qui placent la cité sous la dépendance et la menace potentielle des puissances extérieures, soit qu'ils seront 'libres vis-à-vis de leurs appétits', appétits dont a vu que le commerce avait tendance à les attiser. La suite du texte rend ce second sens plus probable. Ce qui est en effet exclu de la sphère législative, ce sont des pratiques en très grand

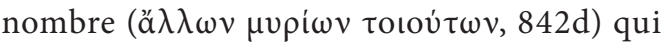
partagent toutes la caractéristique de ne pas fournir directement le nécessaire mais de procurer de l'argent à la cité et aux individus 
en vue de l'achat du nécessaire: sans quoi on ne comprendrait pourquoi l'Athénien évoque l'hôtellerie et les mines dans cette liste. ${ }^{18} \mathrm{Si}$ les activités commerciales sont sans doute celles qui y parviennent le mieux - l'Athénien en nomme quatre formes, l'hôtellerie incluse - ce ne sont toutefois pas les seules. La méfiance de l'Athénien à leur sujet relève d'une méfiance plus générale portant sur le désir d'argent et les pratiques économiques auxquelles il donne lieu, qui risquent d'ouvrir la porte à la démesure et à l'excès. ${ }^{19}$ C'est ce que confirmera l'Athénien peu après en interdisant non pas le commerce de détail mais le 'commerce de détail en vue de faire des affaires'

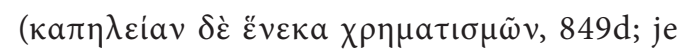
souligne). Tous ces éléments invitent donc à nuancer l'hostilité envers le commerce prêtée à Platon sans plus de précision.

Malgré le refus d'une économie placée sous le signe de la médiation financière, les lois sur le commerce et l'argent font leur apparition dans la future colonie parce que la parfaite autosuffisance est à peu près impossible: si le territoire 'ne manque d'à peu près

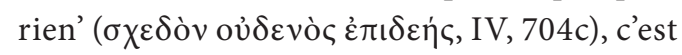
qu'il manque de certaines choses. ${ }^{20}$ Comment alors introduire le commerce dans la cité sans faire le jeu du désir d'enrichissement? Tout l'effort de l'Athénien consiste à le maintenir dans ses marges.

\section{LE COMMERCE DANS LES MARGES DE LA CITÉ}

La législation de l'Athénien autour du commerce répond à trois questions: quels biens peuvent faire l'objet du commerce? Qui peut commercer? Quelle forme doit prendre le commerce? L'ensemble de toutes ces prescriptions vise à le rendre le plus extérieur possible à la cité tout en l'y acceptant dans ses marges et sous certaines conditions.

\section{Les objets du commerce}

Les interdictions relatives au commerce portent principalement sur tout ce qui met en péril l'intégrité et l'unité de la cité. Deux sont fondamentales de ce point de vue. La première concerne les lots, c'est-à-dire les parcelles de terrain que les citoyens ont reçues pour y vivre et les exploiter. Il est interdit de 'déprécier le niveau et l'importance des possessions imparties à l'origine [aux citoyens] selon la juste mesure, par des transactions mutuelles d'achat et de vente' ( $\tau \tilde{\omega} \tau \tau$

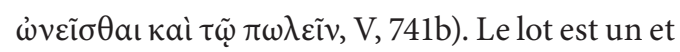
indivisible, et il sert l'égalité arithmétique entre les citoyens parce qu'un principe d'égalité dans leur rendement a été institué dès le départ, leur taille étant fonction de leur fertilité (V, 745c-d). En vendre ou en acheter une partie contreviendrait à ce principe. À plus forte raison, le lot lui-même est inaliénable et ne peut être ni acheté

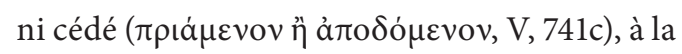
fois parce que la cité s'identifie à son territoire, que les lots ne sont pas la propriété des citoyens mais la propriété commune de la cité (V, 740a), et que le nombre de 5040 lots a été déterminé pour répondre à des exigences de proportion et de distribution (V, 737e-738a). De manière plus radicale encore, aucun des citoyens investis d'un lot ne pourra devenir 'ni marchand détaillant

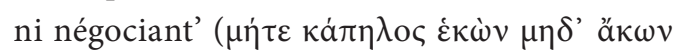

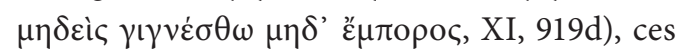
deux activités risquant de compromettre celle du citoyen et par là l'intégrité de la cité.

La seconde interdiction concerne les importations et les exportations, avec cette différence par rapport aux transactions portant sur le lot qu'il ne s'agit plus d'interdire les exportations et les importations, mais de les limiter selon le double critère de ce 
qui est nécessaire ou non-nécessaire pour l'intégrité et l'autosuffisance de la cité, et toujours dans l'idée de restreindre le contact avec l'étranger. Il est interdit d'importer ce qui n'est pas nécessaire - l'exemple le plus précis est celui des encens 'venant de l'étran-

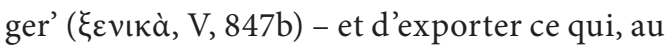
contraire, est nécessaire à la cité (VIII, 847b-c).

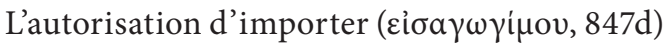
tout ce qui sert à fabriquer des armes et des instruments indispensables à la guerre n'est pas signalée parce que ces importations seraient les seules permises, ${ }^{21}$ - l'Athénien a mentionné juste avant les importations en général (847b) - mais parce que, destinées à assurer la défense de la cité, elles sont un cas particulièrement important de ce qui lui est nécessaire (VIII, $847 \mathrm{c}-\mathrm{d}$ ), et parce qu'elles doivent se faire pour cette raison dans des conditions précises: elles sont placées sous la direction des commandants de cavalerie (les hipparques) et des stratèges, qui sont 'responsables des importations et des exportations' de ce genre (

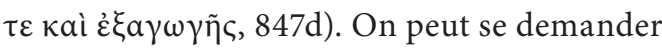
pourquoi, dans ce passage, l'Athénien mentionne aussi les exportations alors qu'il vient juste avant de parler uniquement des importations de ce qui est nécessaire à la guerre. L'explication se trouve dans la partie suivante de la phrase - 'la cité donnant et recevant en

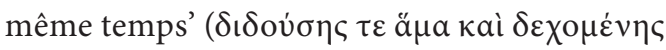
$\tau \tilde{\eta} \varsigma \pi$ ó $\lambda \varepsilon \omega \varsigma, 847 \mathrm{~d})$ - qui renvoie à cette idée que la cité ne peut obtenir ce dont elle a besoin de l'extérieur sans proposer en retour ce dont pourrait avoir besoin la cité dont elle importe les produits. C'est ainsi que dans la République Socrate décrit le lien indissociable des importations et des exportations, et qu'il explique la fonction des marchands. ${ }^{22}$ Les chefs militaires devront donc veiller à la fois à ce que soit importé ce qui est nécessaire pour la guerre, et à ce que ne soient pas exportées les denrées locales également nécessaires à la défense de la cité. Leur connaissance dans ce domaine leur donne la compétence légitime pour s'occuper de ce type de commerce.

Reste le commerce de détail, qui lui aussi obéit au double critère du nécessaire et du nonnécessaire. 'Les peaux, les vêtements en général, le tissu, la laine et les autres produits du même genre [i.e. produits par les agriculteurs] [...], le froment, l'orge destiné à la farine et tout le reste de la nourriture' ne peuvent être vendus aux citoyens ou à leurs esclaves (849c): ces denrées leur étant nécessaires, ils doivent (et, par hypothèse, peuvent) les produire eux-mêmes. Les artisans et leurs esclaves ayant toutefois eux aussi besoin de ces denrées, ils les achèteront à des étrangers, qui eux-mêmes auront acheté au préalable le tiers d'un douzième des produits tirés du sol et des animaux qui leur auront été vendus par les intendants des citoyens (847e-848a). De même avec les produits mentionnés dans liste précédente: l'étranger les achète aux intendants, donc en gros, et les revend au détail aux artisans et à leurs esclaves (849d). La distance que l'Athénien cherche à maintenir entre les citoyens et le commerce de détail ( $\kappa \alpha \pi \eta \lambda \varepsilon i a v, 849 \mathrm{~d})$ est notable ici dans les étapes de ces transactions: les intendants

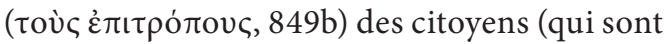
des esclaves ou des étrangers) vendent en gros aux étrangers ce dont ces derniers ont besoin, et ces étrangers à leur tour vendent au détail aux artisans et à leurs esclaves ce dont ces derniers ont besoin (849d), ces artisans étant ceux qui travaillent dans chaque bourg au service de l'exploitation des lots dont les citoyens ont la responsabilité. Reste toutefois un inévitable mouvement d'achat, portant sur 'tous les autres articles et ustensiles dont chacun

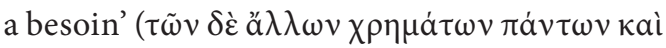

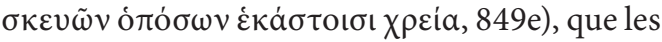
intendants se procurent auprès des étrangers 
pour eux et les citoyens. ${ }^{23}$ Ici, l'Athénien n'a probablement pas en vue le commerce de détail, qui suit un achat en gros, mais le commerce à l'unité d'objets produits en petites quantités.

Loin d'être interdit avec l'extérieur et à l'intérieur, le commerce est plutôt très encadré pour ce qui est de son contenu, au nom d'exigences éthiques et politiques. C'est le cas aussi concernant ceux qui sont autorisés à commercer.

\section{Le commerce: l'affaire des non citoyens}

La circulation et l'acquisition des biens nécessaires dans la cité des Lois sont soumises à un double régime, qu'on pourrait résumer de la façon suivante: on partage dans la cité, on commerce à ses frontières. L'intérieur et les frontières de la cité désignent ici non seulement des lieux (notamment, on va le voir, la place du marché) mais aussi et d'abord des statuts: il y a d'un côté, ceux qui font la cité comme entité politique, à savoir les citoyens et, secondairement, leurs esclaves; et, de l'autre, ceux qui contribuent seulement à la faire: les artisans, leurs esclaves, et les étrangers. Ils ne sont pas vraiment de la cité sans lui être totalement extérieurs non plus.

Ce double régime est explicite dans un passage du livre VIII, dans lequel l'Athénien recommande d'organiser le partage de la nourriture ( coutume 'crétoise', dont il vante la justesse (847e): dans chacun des douze secteurs qui composent la cité ( $\mathrm{V}, 745 \mathrm{~b}-\mathrm{e})$, le total des produits tirés du sol, des produits saisonniers et des animaux sera divisé en trois parts. L'une est pour les citoyens, une autre pour leurs serviteurs, et la troisième est

destinée aux artisans et en général aux étrangers, qu'il s'agisse de métèques, qui habitent avec les citoyens et qui ont besoin qu'on leur fournisse une nourri-

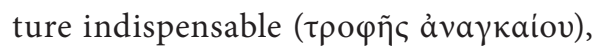
ou de tous ceux qui arrivent jour après jour à la demande de la cité ou de tel ou tel particulier [...]: cette troisième part sera nécessairement la seule à être

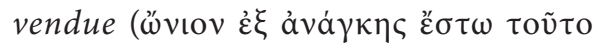
$\mu$ óvov); des deux autres parts, rien ne sera autorisé à être vendu ( $\tau \tilde{\omega} \nu \delta \dot{\varepsilon} \delta v ́ o \mu \varepsilon \rho \tilde{\omega} v$

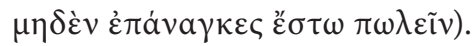

\section{Lois VIII, 848a-b (je souligne)}

On partage avec les siens, on commerce avec les autres. ${ }^{24}$ Les relations avec ces derniers étant inévitables, c'est la seule façon de maintenir en dehors de la cité au sens politique du terme le 'tiers exclu' que forment les étrangers. Le commerce commence là où la politique s'arrête, avec cette nuance toutefois que ce commerce est encadré par des mesures législatives dont la finalité est proprement politique: garantir l'intégrité de la cité et des citoyens.

Cette extériorisation du commerce, ou sa relégation aux frontières de l'espace politique est cohérente avec le fait qu'il doit être l'affaire des étrangers pour ce qui est de la vente au détail ( $\xi \dot{\varepsilon} v o \varsigma[..] \pi \omega \lambda \varepsilon i \tau \omega$, VIII, $849 d)$. Il doit l'être parce qu'il est potentiellement dangereux pour les mœurs et pour la cité: toute vente au détail étant en fait la revente d'un produit acheté en gros, c'est là que la tentation et la possibilité d'un bénéfice important se présentent le plus, que l'économie des médiations financières peut œuvrer contre la cité et ses membres. C'est pourquoi l'une des mesures les plus saisissantes pour limiter le désir de richesse dont le commerce de détail porte la menace consiste à 'n'assigner cette profession qu'à des gens dont la 
corruption morale ne cause pas un grand dommage à la cité' (XI, 919c): les étrangers, par définition extérieurs à la polis, sont donc tout désignés. Il faut certes qu'ils soient en contact avec des membres de la cité mais, on l'a vu, ce ne sont pas directement les citoyens qui se chargent de leur vendre le tiers de la production mais les intendants, qui sont des esclaves et n'exercent donc pas de rôle politique. Il est fort probable que même dans le cas des importations et des exportations relatives à la guerre mentionnées plus haut (VIII, 847c-d), ce ne sont pas les stratèges qui procèdent directement aux échanges, mais leurs esclaves. De même avec le bois que les intendants des campagnes ( $\tau \tilde{\omega} v \dot{\varepsilon} v$

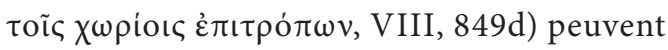
vendre aux étrangers. En outre, cette vente du tiers de la production se fait en gros, non au détail, ce qui limite les risques de réveiller le désir d'enrichissement chez ces intendants. Dans tous les cas, ce ne sont pas les citoyens qui s'en occupent, et pour le commerce de détail, le plus dangereux, seuls les étrangers peuvent s'en charger et vendre aux artisans et à leurs esclaves, et de façon très marginale, les intendants. On notera que ces trois derniers groupes peuvent seulement acheter au détail, pas vendre, ce qui les préserve de la tentation de l'enrichissement inhérente au commerce de détail.

\section{Espaces et temps du commerce}

Une telle relégation du commerce de détail aux frontières de l'espace politique au sens abstrait de cette expression se traduit aussi, sur le plan matériel, dans les lois qui régissent l'installation et le déroulement des marchés dans l'espace et le temps. Commençons par l'espace: celui-ci est organisé de telle sorte que les citoyens et ceux qui leur servent d'intendants ne soient jamais en contact avec la vente de détail, que ce soit pour vendre ou pour acheter. L'espace commercial est en effet fondé sur la séparation entre ce que les intendants des citoyens vendent en gros aux étrangers, et ce que les étrangers vendent ensuite au détail aux artisans et à leurs esclaves, à quoi semblent correspondre respectivement deux sortes de places du marché. Il y a d'abord celle qui se situe dans chacun des douze villages (VIII, $848 \mathrm{~d}$ ). C'est là que, sous la surveillance des agoranomes chargés de surveiller ce qui touche à la

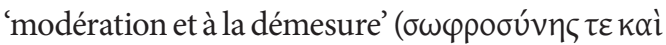
ü $\beta \varepsilon \omega \varsigma, 849 a)$, les intendants des citoyens vendent aux étrangers la part de la récolte qui leur est réservée, ainsi que d'autres produits. Or la vente au détail de ces produits est interdite sur cette place (849c). Pour pouvoir revendre au détail ces produits aux artisans et à leurs esclaves, les étrangers devront se rendre sur le second type de place du marché, exclusivement dédiées à ces transactions et aux étrangers $(\dot{\varepsilon} v \delta \dot{\varepsilon} \tau \alpha \tilde{i} \varsigma \tau \tilde{\omega} v \xi \xi \dot{\varepsilon} v \omega v$

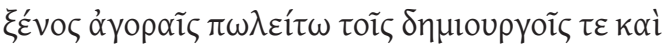

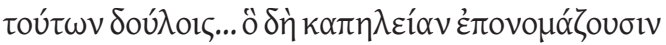
oi $\pi \lambda \varepsilon \tilde{\tau} \sigma \tau 0 \mathrm{l}, 849 \mathrm{c}-\mathrm{d})$. La répétition du commerce de détail dans ces lignes indique qu'il représente le péril le plus grand pour la cité et ses citoyens.

Au souci de la différenciation spatiale s'ajoute un souci de transparence, qui se traduit dans le fait que les autres 'articles et ustensiles' (849e) vendus et achetés seront placés à des endroits précis de la place du marché, endroits déterminés par les gardiens des lois et les agoranomes (849e). Ici, il est probable que l'Athénien n'ait pas en vue le commerce de détail, qui suit un achat en gros, mais le commerce à l'unité d'objets produits en petites quantités. En outre, il évoque sans doute le premier type de place du marché, s'il est vrai que ces 'articles et ustensiles' sont ceux 'dont chacun a besoin' (849e), y compris donc les citoyens. ${ }^{25}$ C'est ce que semble confirmer un passage du livre XI, selon lequel 'tout échange qui se fait par achat ou par vente entre 
une personne et une autre se fera par livraison à la place assignée pour chaque article ( $\varepsilon \vee \chi \omega ́ p \alpha$

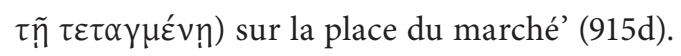
Dans tous les cas, assigner un lieu aux objets des transactions, c'est s'assurer d'un contrôle plus efficace sur ce qui est vendu et en quelle quantité, ce qui n'est pas étranger, on le verra, aux enjeux de vérité que Platon place dans le commerce.

En ce qui concerne l'organisation temporelle des transactions commerciales, elle présente deux aspects. Le premier porte sur le calendrier mensuel de ces transactions, que l'Athénien établit de la façon suivante. Le ler de chaque mois sera vendue aux étrangers leur part de blé pour un mois. ${ }^{26}$ Le 10 de chaque mois, on leur vendra les liquides pour un mois (sans doute le vin et l'huile). Enfin, le 23 de chaque mois on leur vendra les animaux et tous les objets et instruments qu'ont à vendre les agriculteurs (comme les peaux ou les tissus) (VIII, 849b-c). Cette séparation dans le temps et cette mensualité des ventes des différents produits nécessaires a plusieurs effets. Elles s'opposent clairement à l'institution du marché quotidien, qui devait être la règle à Athènes, et obligent ainsi à la modération et à la régularité dans la consommation, laquelle est souvent marquée par l'excès et propice à une spéculation de la part des (re) vendeurs. Le second aspect temporel des transactions commerciales sur lequel la loi se prononce concerne le temps de l'échange lui-même: il doit avoir lieu au moment même où le prix est payé, où la monnaie et la denrée changent de mains

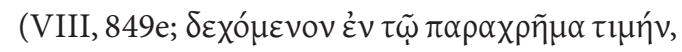
XI, 915d). L'interdiction de la vente et de l'achat à crédit (XI, 915d-e), et le fait que la loi restera sourde à toute réclamation de celui qui s'estime lésé dans le cadre de pratiques formellement similaires au crédit - parce qu'il n’a pas reçu la marchandise qu'il a payée d'avance ou parce qu'il n'a pas reçu le prix de la marchandise qu'il a livrée d'avance (VIII, 849e-850a) - signalent bien que le présent de la transaction est un moment décisif de vérité éthique et de justice. Pour anecdotique et prosaïque qu'il paraisse, son enjeu dépasse le simple échange matériel: il engage la valeur infinie de la justice et de la vérité dans l'échange fini et répété des biens et de la monnaie. Que la transaction doive avoir lieu au présent ne signifie pas qu'elle n'engage pas l'avenir: au contraire, elle doit l'assurer, précisément parce qu'elle aura eu lieu dans les meilleurs conditions morales et, par suite, politiques. Qu'il s'agisse de l'espace ou du temps, l'organisation rigoureuse du commerce vise donc un seul et même objectif: donner la plus grande mesure et la plus grande régularité à des activités qui sont le lieu ordinaire de l'excès, du mensonge et de l'injustice. C'est ce que signale aussi la relative positivité morale et politique que l'Athénien reconnaît au commerce, sous certaines conditions, vers la fin des Lois.

\section{JUSTICE ET VÉRITÉ: LE SENS DU COMMERCE}

De la transparence à la vérité

Pour l'Athénien, l'importance de la vérité et de la justice dans le commerce est d'abord négative ou indirecte: il s'agit, en légiférant sur les transactions commerciales, de mettre à distance tout ce qui pourrait faire obstacle à ces deux valeurs. C'est pourquoi il propose une série de lois et de châtiments contre tout ce qui s'apparente à une forme de fraude ou de dissimulation dans les transactions commerciales, en accord avec le souci de transparence évoqué plus haut à propos de l'organisation spatiale du commerce. Ainsi pour toute vente réalisée supérieure à 50 drachmes, quand il s'agit d'esclaves, le vendeur sera tenu de rester en ville pendant dix jours à partir de la date de la vente, pour que l'acheteur ait la possibilité de lui retourner la marchandise sous 
certaines conditions qui mettent toujours en jeu la connaissance, de la part de l'acheteur comme du vendeur, de la qualité du 'produit': par exemple si l'esclave est porteur d'une maladie qui n'est pas immédiatement visible, ou s'il a commis un crime (XI, 915e-916d). Si, au moment de l'achat, l'acheteur sait que l'esclave est malade parce que le vendeur le lui a dit ou parce qu'il est lui-même médecin ou maître de gymnastique, la transaction ne peut être annulée; de même s'il sait que l'esclave a commis un crime. Mais tout défaut de connaissance, qu'il soit involontaire de la part de l'acheteur comme du vendeur, ou qu'il résulte d'une dissimulation de la part du vendeur, rendra possible le retour de l'esclave au vendeur, avec des sanctions diverses pour ce dernier. De manière générale et somme toute classique, le commerce ne doit pas nuire à la vérité, et par là à la justice et à la paix dans la cité, comme le confirme l'interdiction générale de vendre quoi que ce

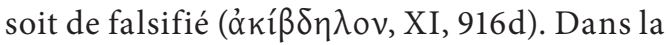
loi sur la fraude qui suit immédiatement ce passage, la section consacrée aux punitions (917b-918a) montre que ce sont les transactions commerciales que l'Athénien a particulièrement en vue depuis le préambule (916d917b), même si elles ne sont pas nommées comme telles. Toute tentative de falsification ou de tromperie relève du même genre que le

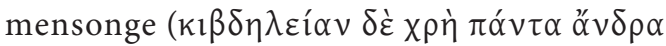

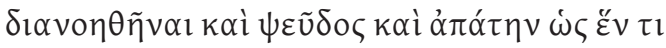
үદ́voৎ őv, 916d), les trois formes du faux commercial étant qu'un même objet soit annoncé à deux prix distincts le même jour (917b-c), que l'on vante l'objet à vendre (917c), et que l'on vende une marchandise falsifiée (917ce) ${ }^{27} \mathrm{~S}$ y livrer en prenant les dieux à témoins, c'est les outrager (916e-917a), eux qui ont des sanctuaires sur chacune des douze places de marché (VIII, 849a).

\section{Éloge du commerce}

Mais avec une telle loi, l'Athénien ne se contente pas de sanctionner ce qui, dans le commerce, peut nuire à la vérité et à la justice, il propose aussi de libérer ce qui, dans ces mêmes transactions, leur est favorable. La preuve en est fournie par l'éloge que l'Athénien fait du commerce de détail, à condition qu'il soit pratiqué par des gens honnêtes (XI, 918d-e). Dans ce passage qui n'est pas sans rappeler l'importance que Socrate accorde aux transactions commerciales de détail dans la naissance de la cité au livre II de la République (371b-d), l'Athénien montre que ce n'est pas le commerce qui corrompt les hommes mais les hommes qui corrompent le commerce, qui le dénaturent. La kapeleia n'a pas pour but de nuire, sa fonction naturelle est au contraire d'introduire, grâce à la monnaie, uniformité et proportion entre choses qui en sont dépourvues (918b-c). Ce sont des procédés malhonnêtes visant l'enrichissement et contraires à la philia 'qui ont jeté le discrédit sur ces pratiques' (919b). Activité tendanciellement sophistique qui fait passer le faux pour le vrai dans le but d'en retirer le plus grand profit, le commerce n'est donc pas condamné à être pratiqué de la sorte. Dans le commerce de détail tel que l'institue l'Athénien se joue une part positive de l'armature morale et politique de la cité. Ce commerce, si limité soitil dans la future colonie, est la forme concrète et élémentaire de la relation éthique et politique que l'Athénien place à la base de la cité: il est, à même l'échange des biens et de l'argent, un exercice en acte de la vérité et de la justice.

\section{CONCLUSION}

Que l'Athénien évoque là une pratique idéale ou rêvée du commerce, c'est ce dont témoignent les trois remèdes qu'il envisage pour limiter ses dangers pour la cité: avoir le plus petit nombre possible de marchands de détail (kapèlôn), n'as- 
signer à cette profession que ceux dont la corruption ne cause pas de tort à la cité, et travailler à éviter la dégradation morale de ces marchands (919c). On notera qu'avec ce dernier remède, il nuance le pessimisme anthropologique qui se dégage des deux autres.

\section{BIBLIOGRAPHIE}

J. Alvey, A Short History of Ethics and Economics, Edward Elgar, Cheltenham 2011.

É. Benveniste, Le Vocabulaire des institutions indoeuropéennes, vol. 1, Minuit, Paris 1969.

A. Bresson, La Cité marchande, De Boccard, Paris 2000.

É. Helmer, La Part du bronze. Platon et l'économie, Vrin, Paris 2010.

J. Lacourse, 'Réciprocité positive et réciprocité négative: de Marcel Mauss à René Girard’, «Cahiers Internationaux de Sociologie», NS, Vol. 83 (1987), 291-305.

P. Karvonis, 'Le vocabulaire des installations commerciales en Grèce aux époques classique et hellénistique', in J. Andreau, V. Chankowski (dir.), Vocabulaire et expression de l'économie dans le monde antique, De Boccard, Paris 2007, 35-50.

L. Kurke, 'Kapèleia and Deceit: Theognis 59-60', "The American Journal of Philology», Vol. 110, N4 (1989), 535-544. http://www.jstor.org/ stable/295278

R. Mayhew, Aristotle's Criticism of Plato's Republic, Rowman \& Littlefield, Lanham 1997.

M. Piérart, Platon et la cité grecque. Théorie et réalité dans la constitution des Lois, Académie Royale de Belgique, Bruxelles 1974.

S. Sauvé-Meyer, 'Les dangers moraux du travail et du commerce dans les Lois de Platon', «Revue Française d'Histoire des Idées Politiques», $\mathrm{N}^{\circ} 16,2$, 2002, 387-397.

K. Schöpsdau, Platon Nomoi (Gesetze), Übersetzung und Kommentar, Vandenhoeck \& Ruprecht, Göttingen, 1994-2011.

A. Schriefl, Platons Kritik an Geld und Reichtum, De Gruyter, Berlin, 2013.

R.F. Stalley, An Introduction to Plato's Laws, Blackwell, Oxford 1983.

J.I. Weinstein, 'The Market in Plato's Republic', «Classical Philology», Vol. 104, N4, (2009), 439-458 http://www.jstor.org/stable/10.1086/650979

\section{NOTES}

1 Cet article a été rédigé dans le cadre d'un projet de recherche financé par une bourse d'été ('summer stipend') offerte par le National Endowment for the Humanities (USA) durant la période juin-juillet 2015.

2 Stalley 1983, 101 et 103; Baeck 1994, 69-70; Alvey 2011, 79. Voir Leg. IV, 704a-705c; XI, 918e-919b. Cf. Rep. IV, 435e-436a.

3 Sauvé-Meyer 2002.

4 Sauvé-Meyer 2002, 388; 390.

5 Benveniste 1969, 140.

6 Karvonis 2011.

7 Rep. VIII, 557c; 558c.

8 Hérodote, Histoires I, 153: 'Cyrus demanda aux Grecs qui étaient présents quelle sorte d'hommes c'était que les Lacédémoniens [...]. Sur la réponse qu'ils lui firent, il parla ainsi au héraut des Spartiates : “Je n’ai jamais redouté cette espèce de gens qui ont au milieu de leur ville un endroit $(\chi \tilde{\omega} \rho \circ \zeta)$ où ils s'assemblent pour se tromper les uns les autres par des serments réciproques [...]." Cyrus lança ces paroles menaçantes contre tous les Grecs, parce qu'ils ont dans leurs villes des places où l'on vend

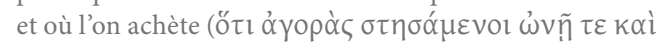
$\pi \rho \eta ́ \sigma l ~ \chi \rho \varepsilon ́(\omega \nu \tau \alpha l)$, et que les Perses n'ont pas coutume d'acheter ni de vendre ainsi dans des places ( $\alpha$ үo

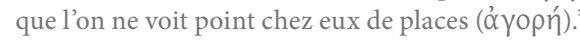

9 Kurke 1989, 540.

10 Soph. 222b-231e; Hipp. mai. 281a-282e. Voir Helmer 2010, 93-98.

11 Toutefois, l'éloge qui repose sur un savoir vrai possède une force éducative et politique: par exemple Leg. VII, 801 è propos des morts de la cité.

12 Sur l'importance de la philia dans la cité, voir Rep. III, 386a; Leg. VIII, 836e-837a.

13 À l'époque classique, Athènes, au contraire, recourt massivement aux importations pour la nourriture, notamment le blé. Platon semble répondre ici de très près à un passage de l'Histoire de la Guerre du Péloponnèse II, 38, 2: 'Nous voyons arriver chez nous, grâce à l'importance de notre cité, tous les produits de toute la terre, et les biens fournis par notre pays ne sont pas plus à nous, pour en jouir, que ne sont ceux du reste du monde'

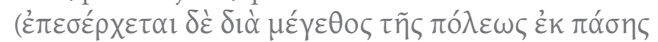

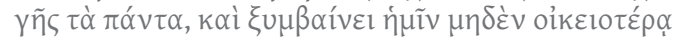

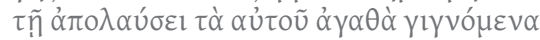

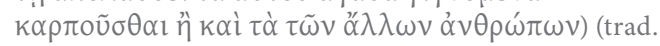
J. De Romilly, Paris, Les Belles Lettres, 1962).

14 Leg. III, 695e-696b.

15 Rep. I, 343b-344c; IV, 422d-423b. Voir aussi Leg. V, 742d. 16 Voir la note 13.

17 Histoire de la guerre du Péloponnèse I, 6-17. Voir aussi Y. Garlan, 'Signification historique de la piraterie grecque', «Dialogues d'histoire ancienne», Vol. 4 (1978), 1-16.

$18 \mathrm{Au}$ contraire du projet d'exploitation des mines du Laurion présenté par Xénophon dans les Revenus pour procurer des revenus monétaires à Athènes.

19 D'autres mesures le confirment: l'interdiction de 
toute espèce de droits à acquitter pour importer ou exporter (VIII, 847b), ou le choix d'une monnaie à usage strictement local et l'interdiction de posséder des devises étrangères $(V, 742 \mathrm{a}-\mathrm{c})$.

20 Même idée en Rep. II, 370e.

21 Contrairement à ce que soutiennent Piérart 1974, 251; et Bresson 2000, 116.

22 'Socrate - Mais si celui qui est chargé [de trouver à l'extérieur ce dont sa cité a besoin] s'en va les mains vides, n'apportant rien de ce dont manquent ceux chez qui on voudrait se procurer ce dont on a soi-même besoin, il reviendra les mains vides, n'est-ce pas?', Rep. II, 371b. Voir Bresson 2000, 118.

23 C'est en ce sens que j'interprète Ékó $\sigma \tau 0 l \sigma l$.

24 La notion de partage ou de distribution est omnipré-

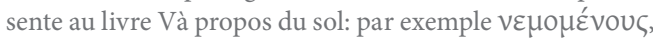

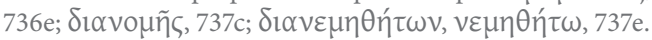
25 Voir la note 23.

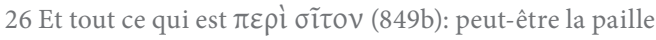
et le chaume?

27 Sur l'éloge, voir plus haut p. 5. 